\section{Determinación cuantitativa de la capacidad antioxidante presente en las semillas de ahuyama (cucurbita máxima) hortaliza típica del Quindío}

\section{Quantitative determination of antioxidant capacity found in ahuyama seeds (cucurvita máxima), a vegetable typical of the Department of Quindio}

\author{
Irma María García Giraldo*, Henry Reyes Pineda** \\ *Químico Puro. Universidad del Quindío. Facultad de Ciencias Básicas y Tecnológicas. Grupo Químico en \\ Investigación y Desarrollo Ambiental. \\ ** Ph.D Ingeniería Química y Nuclear .Decano Facultad de Ciencias Agroindustriales. Universidad del \\ Quindío. Grupo Químico en Investigación y Desarrollo Ambiental.
}

\section{Resumen}

En la actualidad la importancia de los antioxidantes como las vitaminas A, E y C aumenta su, relevancia por los efectos que tienen en la salud humana. Teniendo en cuenta esto, la investigación fue realizada con muestras de semillas de ahuyama en la etapa de pos-cosecha en el municipio de Armenia, Quindío, efectuándose la caracterización físico-química: Potencial de hidrógeno $(\mathrm{pH})$, sólidos solubles $\left({ }^{\circ} \mathrm{Brix}\right)$, actividad de agua (aw), color y acidez. Se utilizó la técnica de ABTS para hallar la capacidad antioxidante de esta especie.

Palabras clave: Ahuyama, ABTS, capacidad antioxidante, vitamina E.

\begin{abstract}
Current importance of antioxidants, such as $\mathrm{A}, \mathrm{E}$ and $\mathrm{C}$ vitamin, becomes more important due to their effect on human health. Bearing in mind this fact, the research was carried out using samples of ahuyama seeds, in the postharvest stage, in the municipality of Armenia, Quindio. A physic-chemical characterization is made. Hydrogen potential $(\mathrm{pH})$; soluble solids ( ${ }^{\circ}$ Brix), water activity (aw), color and acidity. ABTS technique was used to find the antioxidant capacity of this specie.
\end{abstract}

Key words: Ahuyama, ABTS, antioxidant capacity, Vitamin E.

Producto derivado del Trabajo de Grado Maestría en Química. Grupo Químico en Investigación y Desarrollo Ambiental. Categoría B Colciencias. Universidad del Quindío.
Recibido: 02/02/2016

Revisado: $15 / 02 / 2016$

Aceptado: 01/12/2016

Correspondencia de autor:

irmaquimk@hotmail.com hreyes@uniquindio.edu.co

(C) 2016 Universidad La Gran Colombia. Este es un artículo de acceso abierto, distribuido bajo los términos de la licencia Creative Commons Attribution License, que permite el uso ilimitado, distribución y reproducción en cualquier medio, siempre que el autor original y la fuente se acrediten.

Cómo citar:

García, I.M., Reyes, H. Determinación cuantitativa de la capacidad antioxidante presente en las semillas de ahuyama (curubita máxima) hortaliza típica del Quindío. UGCiencia, 22, 193-197.

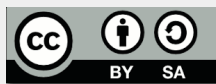




\section{Introducción}

Los antioxidantes son compuestos los cuales pueden inhibir o retardar la oxidación de otras moléculas inhibiendo la iniciación y/o propagación de las reacciones en cadena de los radicales libres (Consumer, E., 2012). Los antioxidantes se dividen en dos categorías principalmente que son: sintéticos y naturales. En general los sintéticos son compuestos de estructuras fenólicas con varios grados de sustitución alquílica, mientras que los naturales pueden ser: compuestos fenólicos (tocoferoles, flavonoides y ácidos fenólicos), compuestos nitrogenados (alcaloides, derivados de la clorofila, aminoácidos y aminas) o carotenoides, así como el ácido ascórbico (Amzad M. 2011). Los antioxidantes sintéticos como el BHAy BHT (Butil - hidroxianisol y Butil - hidroxitolueno) han sido utilizados como antioxidantes desde principios del siglo pasado (Y.S. Velioglu, 1998). Sin embargo, se han impuesto medidas de precaución y se ha restringido su uso dada su carcinogenicidad (Barros et al, 2008). Debido a esto, el interés por los antioxidantes naturales se ha incrementado considerablemente ya que la capacidad de actuar como antioxidante se ha demostrado en el laboratorio y mencionado en la literatura (P. Pietta, 2004).

Los antioxidantes, tales como la vitamina $\mathrm{E}, \mathrm{A}$ y $\mathrm{C}$ actúan para proteger las células contra los efectos de los radicales libres, los cuales son potencialmente dañinos, y pueden dañar las células y contribuir al desarrollo de enfermedades cardiovasculares y cáncer. Estudios se están llevando a cabo para determinar si los antioxidantes a través de su habilidad para limitar la producción de dichos radicales, podrían ayudar a prevenir o retrasar el desarrollo de estas enfermedades crónicas (Molineux, 2004). Existen muchos métodos para medir la capacidad antioxidante de una especie o sustancia, uno muy usado es el del ABTS el cual fue reportado inicialmente por Miller y colaboradores, y se fundamenta en la capacidad de un antioxidante para estabilizar el radical catión coloreado ABTS •+, el cual es formado previamente por la oxidación del ABTS (2,2'-azinobis (3-etilbenzotiazolina-6- acido sulfónico)) por metamioglobina y peróxido de hidrogeno. Los resultados son expresados como equivalentes de Trolox o TEAC (por su nombre en ingles, Trolox Equivalent Antioxidant Capacity) (Brand-Williams, 1997). La ahuyama (cucúrbita máxima), es una baya rica en carotenos y vitaminas tales como la E, A y B, es ampliamente utilizada en el departamento del Quindío para el consumo en diversas preparaciones, sopas, jugos, tortas pequeñas, cremas, quesillo, torta, flan y el tradicional mote de ahuyama. Este proyecto evaluará la capacidad antioxidante de este fruto con el fin de aprovechar un desecho agrícola "semillas", debido a los múltiples beneficios en la salud humana (LA. Bystrom, 2008).

\section{Materiales y métodos}

Para realizar la caracterización físico-química se compraron las muestras de ahuyama en el supermercado Éxito del municipio de Armenia, Quindío; posteriormente se le determinaron los siguientes parámetros a las semillas: actividad de agua (aw), potencial de hidrógeno $(\mathrm{pH})$, grados brix ( ${ }^{\circ}$ Brix), color y acidez titulable. Para cada una de las variables medidas se tomaron tres muestras de forma aleatoria y se dividieron en tres bloques los cuales fueron escogidos completamente al azar.

La actividad de agua se pudo determinar tomando las muestras de pulpa y macerándolas hasta homogenización, se escogieron las mejores semillas y teniendo ya las muestras listas se llevaron al higrómetro a una temperatura de \pm $26,3^{\circ} \mathrm{C}$ y se tomaron las lecturas de las mismas (P.F. Hsu, et al., 2008). En la determinación de grados brix (cantidad de azúcar), se licuó el fruto y semillas, se filtraron y fueron llevadas al refractómetro. 
En cuanto los valores de color, se tomaron muestras de la ahuyama licuadas, se colocaron en el colorímetro y se midieron los parámetros a, 1, b, c y h.

Para la acidez titulable las muestras fueron licuadas y filtradas tres veces de tal manera que las muestras de pulpa y semillas quedaran completamente homogéneas y sin residuos sólidos, antes de realizar la titulación se estandarizó el hidróxido de sodio con biftalato de potasio, después de tener las soluciones de las muestras se les agregaron dos gotas de fenolftaleína y se procedió a la titulación con hidróxido de sodio (R, L., et al., 2013).

El último parámetro, potencial de hidrógeno, las muestras fueron leídas en el pH-metro. Los análisis de capacidad antioxidante se determinaron por la técnica el ABTS las cuales se describen a continuación.

\section{Resultados}

\section{Generación de radical catión ABTS•+.}

Se pesó $77.6 \mathrm{mg}$ del reactivo ABTS y se adicionó $20 \mathrm{~mL}$ de agua destilada para obtener una concentración de $7 \mathrm{mM}$ en solución acuosa. Posteriormente se pesó $13.2 \mathrm{mg}$ de persulfato de potasio $(2.45 \mathrm{mM})$ y se hizo reaccionar en un frasco ámbar con la solución de ABTS•+, dichas soluciones se homogenizaron y se cubrió con papel aluminio. La solución se dejó incubar durante 16 horas a temperatura ambiente; por último, se diluyó la solución de ABTS•+ en etanol absoluto hasta obtener una absorbancia inicial de $0.7 \pm(0.02)$ a $732 \mathrm{~nm}($ Skoog, D et al. 1992).

\section{Preparación de las muestras de ahuyama}

La determinación de la actividad antioxidante de los extractos vegetales se llevó a cabo de acuerdo con el método propuesto por (Brand-Williams B., 1997), con ligeras modificaciones como se describe a continuación. Cada ensayo se realizó
3 veces y a su vez cada muestra por triplicado (Murillo, 2004). Se pesaron 5g de semillas de ahuyama, se maceraron y fueron aforados a 25 $\mathrm{ml}$ con etanol absoluto, luego cada una de las muestras fueron llevadas a un agitador recíproco durante una hora, se extrajo el sobrenadante y se llevaron a una centrífuga a 1500rpm durante 20 minutos, se recogió el sobrenadante y se aforó nuevamente a $25 \mathrm{ml}$ con etanol, estos fueron unidos con el precipitado y el mismo procedimiento se llevó a cabo 3 veces más (L., B. et al., 2012).

Cuando ya se tenían los extractos de ahuyama se procedió a determinar su capacidad antioxidante para lo cual se tomaron $50 \mu 1$ de los extractos y se les agregaron $1450 \mu 1$ de ABTS y fueron llevados al espectrofotómetro a una longitud de onda de 732nm (Leubolt, R., 1993). A partir de las absorbancias obtenidas se determinó el porcentaje de actividad antioxidante con la ecuación que se presenta a continuación:

$\%$ Actividad Antioxidante $=\quad$ Abs.I Abs.M/ Abs.I

Donde Abs.I, son las absorbancias iniciales para el reactivo de ABTS y Abs.M son las absorbancias obtenidas para cada una de las muestras de semillas de ahuyama.

\section{Discusión de resultados}

Tabla 1. Análisis fisicoquímicos de las semillas de ahuyama

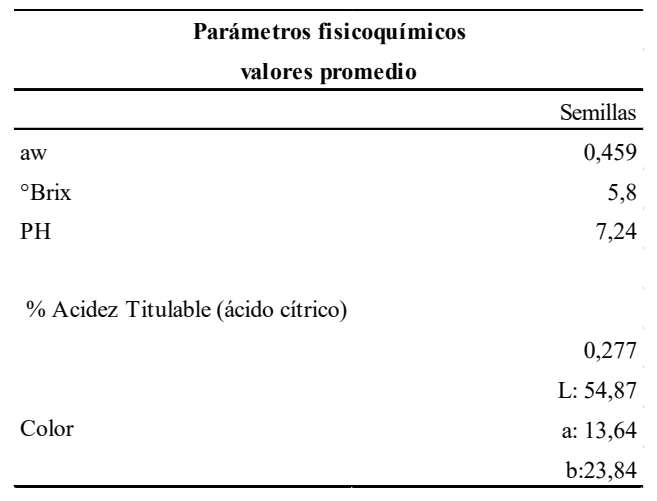

Fuente: Elaboración propia 
En la tabla 1 encontramos todos los valores promedio de cada uno de los análisis fisicoquímicos realizados a las semillas de la ahuyama.

Los parámetros fisicoquímicos evaluados para cada una de las muestras de semillas de ahuyama demuestran que los valores de actividad de agua (aw) obtenidos son bajos y teniendo en cuenta que la aw posee un valor máximo de 1 y un mínimo de 0 , esto nos indica una baja probabilidad de ataque de microorganismos, por lo tanto, este producto se hace más perdurable al momento de su consumo (A.M. Pisoschi, et al.,2010). Los grados brix son los que miden la cantidad de sólidos solubles presentes en un jugo o pulpa expresados en porcentaje de sacarosa. Están compuestos por los azúcares, ácidos, sales y demás compuestos solubles en agua presentes en los jugos de las células de una fruta o verdura, cuando los valores son altos demuestran el grado de madurez y el porcentaje de los componentes mencionados; por lo tanto, los valores obtenidos demuestran que las semillas de ahuyama poseen una cantidad considerable de sólidos solubles y un grado de madurez intermedio. En el caso del $\mathrm{pH}$, el valor promedio indicó tener una acidez baja, lo cual se corrobora al momento de determinar el porcentaje de acidez titulable, cuyos cálculos se realizaron en función del ácido cítrico debido a que es el más abundante en esta verdura, con un valor promedio de $0,277 \%$. Los parámetros de color $\mathrm{L}^{*}, \mathrm{a}^{*}$ y $\mathrm{b}^{*}$ en una muestra de alimento, designan: $L^{*}$, la luminosidad ( $0=$ negro y $100=$ blanco); $a^{*}$, el color rojo (positivos) o verde (negativos) y b*, el color amarillo (positivos) o azul (negativos). Los resultados de los análisis del color para las semillas de ahuyama, demostraron que disminuía su luminosidad y tendía hacia el color amarillo, también se pudo observar que había menor síntesis de licopeno debido a la disminución del color rojo ( $\mathrm{F} \& \mathrm{~F}$, 2004).
En la tabla 2 se muestran los porcentajes de actividad antioxidante obtenidos para cada uno de los extractos de semillas de ahuyama evaluados.

Tabla 2. Porcentaje de actividad antioxidante por el método de ABTS

\begin{tabular}{|c|c|c|c|c|}
\hline \multirow{2}{*}{$\begin{array}{l}\text { Semillas } \\
\text { Ahuyama }\end{array}$} & \multicolumn{3}{|c|}{$\%$ Actividad Antioxidante } & $\begin{array}{l}\text { Promedio } \\
\% \text { AA }\end{array}$ \\
\hline & Ensayo 1 & Ensayo 2 & Ensayo 3 & \\
\hline & 97,87 & 98,29 & 98,46 & 98,04 \\
\hline & 98,04 & 98,38 & 98,55 & 98,37 \\
\hline & 98,21 & 98,46 & 98,63 & 98,54 \\
\hline
\end{tabular}

Fuente: Elaboración propia

Los resultados de actividad antioxidante para las semillas de ahuyama por el método de ABTS, mostraron que este subproducto tiene un alto porcentaje de antioxidantes presentes con un valor promedio máximo de $98,54 \%$.

\section{Conclusiones}

La caracterización fisicoquímica para las muestras de semillas de ahuyama en sus estados de pos-cosecha, reflejaron que esta especie posee una actividad de agua intermedia, factor que resulta determinante para la calidad de la verdura. Además los grados Brix permitieron determinar que a pesar de que el producto investigado era una semilla, contenía una cantidad considerable de sólidos solubles disueltos, los demás análisis reflejaron que este subproducto de la ahuyama poseía un grado bajo de acidez y un porcentaje mínimo de síntesis de licopenos.

La actividad antioxidante hallada por el método de ABTS, demostró ser una técnica eficiente, permitiendo de esta manera establecer el porcentaje de capacidad antioxidante en las semillas de ahuyama, corroborando que este subproducto es altamente nutritivo y bueno para el consumo humano. 


\section{Referencias bibliográficas}

Amzad, M. (2015). A study on the total phenols content and antioxidant activity of essential oil and different solvent extracts of endemic plant Merremia borneensis. Arabian Journal of Chemistry 8 (1), pp. 66-71.

Artés, F., Artés-Hernández, F. (2004). Tratamientos postrecolección del tomate fresco. Tendencias e Innovaciones. Tomates. Producción y comercio. España: Ediciones de Horticultura S.L. Reus

Barros, L., Falcão, S., Baptista, P., Freire, C., Vilas-Boas, M., \& Ferreira, I. C. F. R. (2008). Antioxidant activity of Agaricus sp. Mushrooms by chemical, biochemical and electrochemical assays. Food Chemistry, 111, 68-76.

Brand-Williams B., B. W. (1997). Kinetics and Mechanisms of antioxidant activity using the DPPH free radical method. Food Science and Technology. 30, 609-615. Tomado de: file:///E:/Universidad/Downloads/Kinetics $\% 20$ and $\% 20$ Mechanisms $\% 20$ of $\% 20$ Antioxidant $\% 20$ Activity $\% 20$ using.pdf

Bystrom, L (2008). Characterisation of phenolics by LC-UV/Vis, LC-MS/MS and sugars by GC in Melicocus bijugatus Jacq. Food Chem , 111: 1017-1024. Tomado de: https://www.ncbi.nlm.nih.gov/pmc/articles/ $\underline{\text { PMC3123376/ }}$

Consumer, E. (10 de octubre de 2012). Eroski Consumer. Guia de Hortalizas y Verduras. Tomado de: http://verduras.consumer.es/

Pisoschi, A. M., Danet, A. F., \& Kalinowski, S. (2008). Ascorbic Acid Determination in Commercial Fruit Juice Samples by Cyclic Voltammetry. Journal of Automated Methods and Management in Chemistry. http:// doi.org/10.1155/2008/937651.
Leubolt, R., Klein,H.(1993). Determination of sulphite and ascorbic acid by high-performance liquid chromatography with electrochemical detection. J. Chromatogr. 640, 271-277.

Molineux, P. (2004). The Use of stable free radicaldiphenylpicrilhydrayl for estiming antioxidant activity. Science and Technology, 121: 93-101.

Murillo, J. (2004). Las Euphorbiaceas de Colombia. Biota Colombiana 5(2), 183199. Tomado de: http://www.redalyc.org/ pdf/491/49150203.pdf

Pietta, P. S. (2004). Antioxidant Activity of selected medicinal Plants. Journal of Science and Technology, 211-219.

P.F.Hsu, W. C. (2008). Study poliviologen and the aplication of poliviologen modifed glassy carbon electrode an amperometric detection of vitamin C. Journal of Applied Electrochemistry 38. Tomado de: http://www. springer.com/chemistry/electrochemistry/ journal/10800

Leubolt, R. (1993). Determination of sulphite and ascorbic acid by high-performance liquid chromatography with electrochemical detection. Journal of Chromatography $A$ 640(2). Pp 271-277.

Skoog, D; Holler, F; Nieman, T.(1992). Principios de Análisis Instrumental. . Madrid, España. McGRAW-HILL/ Interamericana de España, S.A.U.

Y.S. Velioglu, G. M. (1998). Actividad Antioxidante y Fenoles totales y frutas selectas vegetales y productos de grano. Journal Agric Food Chem. Tomado de: http://pubs.acs.org/ac$\underline{\text { tion} / \text { doSearch?AllField=Actividad }+ \text { Antiox }}$ $\underline{\text { idante }+\mathrm{y}+\text { Fenoles }+ \text { totales }+\& \text { type }=\text { within } \&}$ publication $=4002602$. 